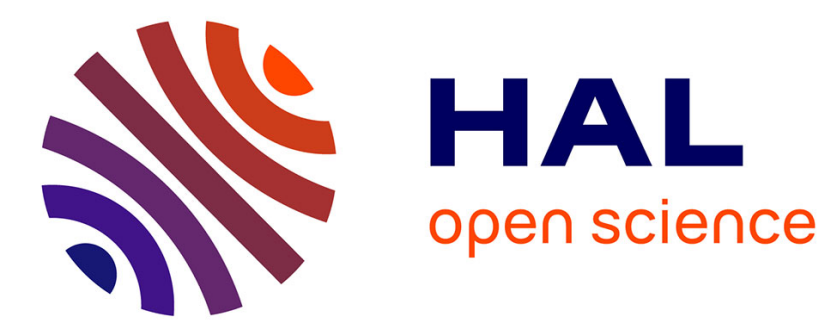

\title{
Political ecology des services écosystémiques
}

\author{
Xavier Arnauld de Sartre, Johan Oszwald, Monica Castro, Simon Dufour
}

\section{To cite this version:}

Xavier Arnauld de Sartre, Johan Oszwald, Monica Castro, Simon Dufour (Dir.). Political ecology des services écosystémiques. PIE Peter lang, 21, 2014, EcoPolis, Marc Mormon, 978-2-87574-197-4. halshs-01098622

\section{HAL Id: halshs-01098622 \\ https://shs.hal.science/halshs-01098622}

Submitted on 27 Dec 2014

HAL is a multi-disciplinary open access archive for the deposit and dissemination of scientific research documents, whether they are published or not. The documents may come from teaching and research institutions in France or abroad, or from public or private research centers.
L'archive ouverte pluridisciplinaire HAL, est destinée au dépôt et à la diffusion de documents scientifiques de niveau recherche, publiés ou non, émanant des établissements d'enseignement et de recherche français ou étrangers, des laboratoires publics ou privés. 


\section{Political ecology \\ des services écosystémiques}

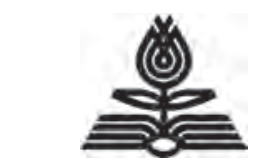

P.I.E. Peter Lang

Bruxelles $\cdot$ Bern $\cdot$ Berlin $\cdot$ Frankfurt am Main $\cdot$ New York $\cdot$ Oxford $\cdot$ Wien 
Ce livre est tiré d'un projet financé par l'agence nationale de la recherche scientifique (France) intitulé Approche géographique des services écosystémiques (ANR JCJC SHS 2010). Il a en outre bénéficié du soutien financier de l'Institut de géographie et durabilité de l'université de Lausanne.

Cette publication a fait l'objet d'une évaluation par les pairs.

Toute représentation ou reproduction intégrale ou partielle faite par quelque procédé que ce soit, sans le consentement de l'éditeur ou de ses ayants droit, est illicite. Tous droits réservés.

(C) P.I.E. PETER LANG S.A.

Éditions scientifiques internationales

Bruxelles, 2014

1 avenue Maurice, B-1050 Bruxelles, Belgique

www.peterlang.com ; info@peterlang.com

Imprimé en Allemagne

ISSN $1377-7238$

ISBN 978-2-87574-197-4

eISBN 978-3-0352-6473-9

$\mathrm{D} / 2014 / 5678 / 73$

« Die Deutsche Bibliothek » répertorie cette publication dans la « Deutsche Nationalbibliografie »; les données bibliographiques détaillées sont disponibles sur le site $<\mathrm{http} / / / \mathrm{dnb}$.ddb.de $>$. 


\section{Table des matières}

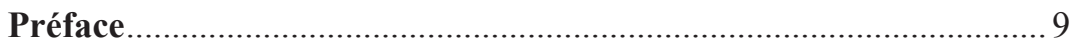

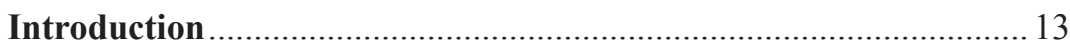

Première Partie. À la recherche du Périmètre de validité DES SERVICES ÉCOSYSTÉMIQUES

Chapitre 1. Modernité écologique et services écosystémiques

Xavier Arnauld de Sartre, Monica Castro, Bernard Hubert et Christian Kull

Chapitre 2. De la biodiversité aux services écosystémiques. Approche quantitative de la généalogie d'un dispositif.

Monica Castro et Xavier Arnauld de Sartre

Chapitre 3. Du MEA à Rio+20 : déploiement et usages

de la notion de services écosystémiques 85

Xavier Arnauld de Sartre, Monica Castro et Denis Chartier

DEUXIÈME PARTIE. QUAND LES SERVICES ÉCOSYSTÉMIQUES DEVIENNENT DES POLITIQUES

Chapitre 4. Quand les réseaux de pro- et d'anti-REDD construisent un outil de gouvernementalité environnementale

Lise Desvallées

Chapitre 5. Les services écosystémiques au Gabon.

Le rendez-vous manqué du renouveau des politiques conservation

Xavier Arnauld de Sartre, Simon Dufour,

Olivier Huet, Johan Oszwald, Noel Ovono Edzang,

Léticia Sello Madoungou

Chapitre 6. Une interprétation brésilienne des Paiements pour services environnementaux. Souveraineté 163 et développement inclusif

Catherine Aubertin, Denis Chartier et Iran Veiga 
TROISIÈME PARTIE. ENJEUX DE LA QUANTIFICATION ET DE LA SPATIALISATION DES SERVICES ÉCOSYSTÉMIQUES

Chapitre 7. Des processus biophysiques aux indicateurs de services écosystémiques. L'apport des approches paysagères

Johan Oszwald, Michel Grimaldi,

Solen Le Clec'h et Simon Dufour

Chapitre 8. Spatialiser des services écosystémiques, un enjeu méthodologique et plus encore 205

Solen Le Clec'h, Simon Dufour, Johan Oszwald, Michel Grimaldi, Nicolas Jégou

Chapitre 9. Cartographie, services écosystémiques et gestion environnementale. Entre neutralité technicienne et outil d'empowerment

Simon Dufour, Xavier Arnauld de Sartre, Monica Castro, Solen Le Clec'h, Johan Oszwald

Conclusion 247

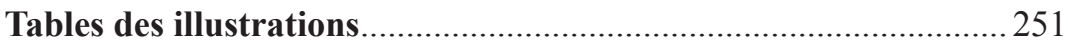

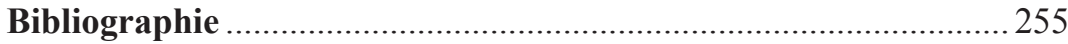

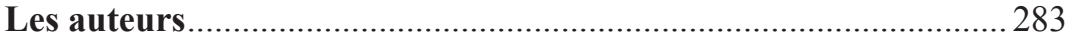




\section{Préface}

La crise de la biodiversité s'observe et s'étudie à des échelles très différentes. Un pédologue peut la décrire à dix centimètres sous le sol, dans la couche humifère qui ne cesse de s'appauvrir du fait de l'intensification agricole, de la déforestation ou encore de la pollution des cours d'eau. Certains écologues s'intéressent à de vastes territoires. Ils recensent et prédisent les extinctions et analysent les impacts biologiques de la dégradation des milieux naturels, grignotés par l'urbanisation, l'agriculture ou les grandes infrastructures. Élevant encore le regard, les climatologues la voient du ciel, observant notre petite « bille bleue » qui s'irrite et s'échauffe. De façon plus métaphorique, la philosophie elle aussi se penche sur le problème d'un point de vue « hors-sol », s'intéressant aux concepts et à leur histoire, interrogeant de haut les rapports HommeNature, les critères impartiaux d'une justice intergénérationnelle ou encore la valeur intrinsèque des entités non humaines. Autant d'échelles, autant de regards, qui enrichissent le caléidoscope de notre compréhension d'un phénomène complexe aux nombreuses facettes. Et pourtant, « la » crise de la biodiversité n'est rien d'autre qu'une multitude de crises imbriquées dans lesquelles se jouent, plus que la vieille adversité entre les humains et la nature, des rapports de force, des mobilisations politiques, des rivalités de personnes et de groupes historiquement et socialement situés. C'est à cette échelle des affaires humaines que s'intéresse ce livre, inspiré d'un champ de recherche dont la traduction littérale du nom en français prête à confusion et que l'on se contente de désigner dans sa version originale : la Political ecology. En posant sur les questions environnementales les regards croisés de la géographie de l'économie politique et de l'anthropologie environnementale dans une posture résolument engagée, la Political ecology offre une ressource précieuse pour analyser la façon dont se configure la « scène " environnementaliste où se croisent scientifiques, gestionnaires, décideurs, militants et entrepreneurs. Car la crise de la biodiversité ne se résume pas à un bouleversement écologique. Elle se traduit par l'apparition d'institutions, de communautés épistémiques et de nouvelles normes inédites.

Parmi ces innovations, la notion de " services écosystémiques » est exemplaire de l'émergence d'un nouveau discours sur les questions environnementales. Définis comme l'ensemble des bénéfices que les êtres humains tirent du fonctionnement des écosystèmes, les services écosystémiques renvoient à une réalité complexe et polymorphe, celle 
de la trame enchevêtrée sur laquelle se nouent les relations entre les communautés humaines, leur environnement et le cortège de vivants non-humains auxquelles elles sont liées de multiples façons : admiration, exploitation, compétition, ignorance, identification, entraide, etc. Tenter d'embrasser une telle diversité de relations et de représentations de façon homogène par la seule notion de " service » relève d'une simplification qui doit éveiller notre vigilance. De façon heuristique, elle peut aider à rapprocher ce qui semblait à première vue distant, offrir un terrain commun pour permettre à des acteurs antagonistes de dialoguer et de construire un projet collectif. Elle peut, en prenant la tangente des conflits frontaux qui opposent bien souvent protecteurs et destructeurs de la nature, devenir une autre façon de considérer nos rapports au monde et l'idée que l'on se fait de ces rapports. Elle peut également ouvrir un véritable chantier interdisciplinaire où se rencontrent enfin sciences humaines et sciences naturelles sans hiérarchie ni asservissement d'une discipline aux autres, mais dans une véritable co-construction d'objets et de savoirs. Voilà certainement le meilleur de ce que peut offrir ce nouveau vocable. Voilà tout le bien que l'on peut espérer de la «mode » des services écosystémiques.

Mais il ne faudrait pas se contenter d'attendre naïvement que le meilleur surgisse de lui-même. Le temps des premiers écologues qui voyaient dans la notion de services écosystémiques un outil essentiellement pédagogique permettant de mettre en évidence la dépendance des sociétés humaines vis-à-vis du support naturel qui les constitue et les maintient est révolu. Ceux qui utilisent et développent aujourd'hui la notion de services écosystémiques y voient véritablement une description du monde. La multiplication, à toutes les échelles, des exercices d'évaluation monétaire de ces services en témoignent. Il ne s'agit plus tant de pédagogie et d'heuristique que d'une requalification de notre rapport à la nature dans un discours à prétention performative qui fait de cette nature un pourvoyeur de biens et de services et qui fait de notre lien à la nature une relation strictement instrumentale, dénuée de désintéressement autant que de réciprocité. La protection de la biodiversité devient essentiellement une question de prudence. Il conviendrait de protéger les forêts tropicales parce qu'elles stockent un carbone qui dérèglerait notre climat s'il était déchargé dans l'atmosphère, de s'abstenir d'assécher les zones humides parce qu'elles fournissent un service de purification de l'eau plus performant que nos usines, de sauver les abeilles domestiques parce que ce sont elles qui pollinisent nos vergers, et de conserver des zones de nature sauvage et diversifiée pour s'y ressourcer et profiter des bénéfices touristiques ainsi engendrés. De là à ce que la protection de la nature ne devienne un business comme les autres, il n'y a plus qu'un tout petit pas à en croire les nombreux promoteurs d'un capitalisme vert, qui entendent 
répondre à la crise actuelle par l'extension des logiques d'ingénierie et de marché à la gestion des milieux naturels.

C'est sur cet étroit sentier qui sépare le pire du meilleur que chemine cet ouvrage, à la fois critique et constructif. En resituant la notion de services écosystémiques dans son contexte d'émergence et de propagation scientifique et institutionnelle, il offre au lecteur un vaste panorama des mutations en cours dans le champ des sciences et des politiques environnementales. D'un côté, il montre les bénéfices d'une approche renouvelée des questions environnementales à travers la mise en évidence des multiples dépendances des systèmes humains vis-à-vis des systèmes naturels. De l'autre, il signale la complexité et les difficultés d'une tentative de rationalisation et de formalisation des liens entre les sociétés et leur environnement à travers la grille d'analyse qu'offre la notion de services écosystémiques. Face au succès fulgurant de l'approche par services dans toutes les sphères de la conservation de la biodiversité - scientifiques, politiques, gestionnaires - une telle analyse est précieuse. Elle permet notamment de dessiner les contours de cette notion et, ce faisant, ne serait-ce qu'en creux ou par défaut, d'en désigner les limites. Car la nature, la biodiversité, les vivants non-humains, s'ils nous rendent des « services », s'ils nous sont « utiles » ou « nécessaires », ne devraient pas être réduits à ce seul statut instrumental. La crise que nous traversons et dont nous sommes responsables nous dit quelque chose d'essentiel sur notre place dans le monde et notre rapport au vivant. Nous sommes dépendants, vulnérables, solidaires. Nous faisons société avec les êtres vivants qui partagent notre espace, constituent et habitent nos champs, nos villes, nos corps. Nous appartenons à une communauté qui excède bien largement les bornes de notre espèce et la conservation de la biodiversité engage notre responsabilité morale au moins autant que notre rationalité prudentielle. Sur cette responsabilité morale, la notion de "services écosystémiques » ne dit rien. Reste à savoir si ce mutisme laissera la place à d'autres discours et à d'autres rationalités ou si au contraire, saturant l'espace des justifications environnementales, l'approche par services ne sera qu'un prolongement et un renforcement des logiques de domination des humains sur la nature qui nous ont conduits à la situation actuelle.

Virginie Maris,

Philosophe, Centre d'Écologie Fonctionnelle et Évolutive 


\section{Introduction}

L'expression " services écosystémiques »a acquis, ces dernières années, une popularité considérable - popularité qui est à la fois la cause et la conséquence de son accession aux forums hybrides de la gouvernance environnementale. Cette expression permet de qualifier les impacts, pour les êtres humains, de crises environnementales majeures : l'érosion de la biodiversité, le changement climatique, l'épuisement des sols, etc. Autant de problèmes globaux qui touchent directement les hommes en ceci que, selon la rhétorique des services écosystémiques, ils menacent la capacité des écosystèmes à maintenir le niveau de services qu'ils rendent aux hommes. On parle ainsi de services climatiques, de services des sols, de service de pollinisation, etc.

L'agitation autour de l'expression " services écosystémiques » est telle que l'on hésite quant au statut que l'on peut lui conférer : s'agitil d'un mot-clef à succès (un buzzword), d'une notion, d'un concept, d'un paradigme ? La question est d'importance, car elle qualifie immédiatement l'objet : le mot-clef à succès renvoie plutôt à une mode, à une opportunité sémantique dont vont se saisir différents acteurs - au premier rang desquels les scientifiques - pour asseoir la légitimité de leurs discours. La notion, elle, a le sens d'idée générale qui, bien que fortement socialisée, reste abstraite dans sa définition : tout le monde s'accorde plus ou moins sur le sens à donner à la notion, sans qu'une définition en soit clairement partagée. En ce sens, elle est plus vague et moins opératoire que le concept, qui définit clairement un objet et sa place dans un corpus théorique plus vaste. Le paradigme renvoie, quant à lui, à une conception théorique dominante qui a cours à une certaine époque dans une communauté scientifique.

Alors, l'expression « services écosystémiques » est-elle le signe d'une mode appelée à passer aussi vite qu'elle est arrivée ? Est-ce plutôt un motvalise qui sert à qualifier la dépendance des hommes aux écosystèmes ? Ou bien doit-on considérer qu'il s'agit de la face émergée d'une théorie des rapports hommes/milieux plus large, voire même l'arrivée d'une nouvelle vision de la nature appelée à structurer profondément les sciences situées à l'interface hommes/natures ? Cette dernière possibilité est sans doute l'objectif, implicite ou explicite, de ceux qui ont institutionnalisé l'expression « services écosystémiques ». Le processus de socialisation et d'apprentissage du discours des services écosystémiques par les acteurs, fussent-ils internationaux ou locaux, a bien pour vocation de changer 
la vision et la relation que ces acteurs développent avec la nature. Ce changement se répercuterait sur la production et la mobilisation des savoirs, qu'ils soient scientifiques, politiques, locaux, Dès lors, il pourra impliquer une requalification des pratiques, des mécanismes d'accès et d'utilisation des ressources, ainsi qu'une réorganisation des structures politiques et sociales en lien direct ou indirect avec les milieux naturels. De nouvelles architectures territoriales pourraient émerger de ce processus.

La question est dès lors autant de s'interroger sur la vocation de l'expression " services écosystémiques » que sur son effet réel - cet effet dépendant fortement de la manière dont elle est appropriée. Ainsi, parler de services écosystémiques dit à la fois beaucoup et peu de la manière dont une société parle de la nature. Cette expression exprime en effet les antagonismes et les enjeux autour de la protection de la nature, et plus particulièrement de son inclusion dans le capitalisme global. Les savoirs, les techniques et les relations de pouvoir des acteurs impliqués se trouvent interrogés par la mise en place de nouveaux dispositifs en créant un dialogue qui mène à la réinterprétation, voire à la reconstruction, de l'expression même et de sa place comme promotrice d'une nouvelle vision de la nature. Si un tel processus ne se met pas en place, si les services écosystémiques en restent au stade de mot-clef, c'est le signe de l'échec d'un projet. À travers l'étude de l'itinéraire de la notion de services écosystémiques, nous voulons comprendre comment les cadres politiques et sociaux participent à la formulation de nouvelles explications à l'interface entre sciences et politique des problèmes environnementaux. Or nous verrons que cet itinéraire n'est ni linéaire ni univoque, que cette expression ne traduit pas un projet délibéré de recadrage des relations hommes/milieux et que son usage dépend fortement des échelles et des acteurs qui s'en saisissent.

Pour avancer dans cette démonstration, et guider les premières étapes de ce travail, nous faisons un pari et un choix. Le pari : considérer qu'en dépit de son usage immodéré par certaines communautés d'acteurs, l'expression " services écosystémiques » est plus qu'un mot-clef. Le changement sémantique qu'elle contient a toutes les chances de marquer durablement les rapports de l'homme à la nature. Le choix : plus qu'un concept, ou même un discours, nous allons parler des services écosystémiques comme un dispositif de pouvoir dans le sens de Michel Foucault (1977), c'est-à-dire comme un ensemble d'éléments hétérogènes qui ont pour vocation de produire des normes, des mouvements de pensée ou des systèmes pour gouverner. Puisqu'il existe une gradation, dans la précision sémantique et l'importance dans la structuration de la pensée, entre la notion, le concept et le paradigme, parlons par défaut de « notion de services écosystémiques » et donnons-nous les moyens de voir s'il y a lieu, dans certains contextes et pour certaines communautés scientifiques, de parler de concept, voire de paradigme. 
Ainsi l'objet de ce livre est-il de comprendre comment cette expression d'origine scientifique a été transformée en un dispositif (dans le sens de Foucault) pour conserver ou gérer la nature et comment ce dispositif agitou pas - dans différents contextes.

\section{L'apparente évidence d'une Notion Frontière}

La notion de services écosystémiques a trois caractéristiques principales qu'il nous importe de préciser : construite sur une métaphore, elle apparaît évidente, naturelle ; son histoire la situe à la rencontre entre science et action; elle est le fruit de la modernité tout en marquant une relative inflexion dans son mode de pensée.

L'évidence de la notion de services écosystémiques s'impose à la lecture de sa définition dans le Millennium Ecosystem Assessment (MEA), rapport commandé par l'Organisation des Nations unies au début des années 2000 , pour qualifier l'état de la biodiversité ${ }^{1}$ : " les services écosystémiques sont les bénéfices que les hommes tirent des écosystèmes » (Millennium Ecosystem Assessment (Program), 2005a). Le MEA a proposé un système de classification des services écosystémiques qui fait référence pour la qualification des services (figure 1).

Figure 1. Qualification schématique des services écosystémiques selon le MEA

\begin{tabular}{|l|l|}
\hline \multirow{5}{*}{$\begin{array}{l}\text { Support } \\
\text { Formation des sols, } \\
\text { Photosynthèse, } \\
\text { Production } \\
\text { primaire, Cycle } \\
\text { nutritif, Cycles de } \\
\text { l'eau }\end{array}$} & $\begin{array}{l}\text { Approvisionnement } \\
\text { Nourriture (culture, élevage, pêche, aquaculture, plantes sauvages } \\
\text { et nourriture animale), Fibres (bois, coton, bois-énergie), } \\
\text { Ressources génétiques, Biochimie et biopharmacie, Eau potable }\end{array}$ \\
\cline { 2 - 2 } & $\begin{array}{l}\text { Régulation } \\
\text { Qualité de l'air, Régulation climatique (globale et régionale/ } \\
\text { de l'eau et traitement des déchets, Régulation des maladies, } \\
\text { Régulation des nuisibles, Pollinisation, Régulation des risques } \\
\text { naturels }\end{array}$ \\
\cline { 2 - 2 } & $\begin{array}{l}\text { Services culturels } \\
\text { Diversité culturelle, Valeurs religieuse et spirituelle, Systèmes } \\
\text { de savoirs, Valeurs éducatives, Inspiration, Valeurs esthétiques, } \\
\text { Relations sociales, Sens des lieux, Valeurs d'héritages culturels, } \\
\text { Loisir et écotourisme }\end{array}$ \\
\hline
\end{tabular}

1 Le titre générique de ce rapport est L'évaluation du millénaire - Millennium Ecosystem Assessment. Dans la mesure où l'expression anglaise est couramment utilisée dans la littérature, nous désignerons ce rapport par ses initiales MEA. 
L'idée au fondement de la notion de services écosystémiques est la suivante : le « bien-être humain » (human well being, expression venue des sciences économiques qui inclut autant les bases matérielles de l'existence humaine que la qualité de vie) dépend de la qualité de son environnement et des ressources qu'il peut en tirer. Ces éléments subissent du fait de l'activité humaine des dégradations massives et rapides inconnues dans l'histoire de l'humanité qui font peser des menaces sur la qualité de la vie sur terre. D'où le fait qu'il faille protéger les écosystèmes pour maintenir les services qu'ils rendent à l'homme.

Cependant, les choix sémantiques effectués pour qualifier les écosystèmes n'ont rien d'évident. Parler de services écosystémiques, c'est associer deux concepts (écosystème et service) renvoyant à des ordres distincts (les milieux naturels et le monde économique) dans une métaphore. Dire que les écosystèmes rendent des services à l'homme, c'est considérer ces écosystèmes en fonction d'un finalisme anthropocentrique (l'homme est la finalité des écosystèmes). La force rhétorique d'une métaphore est alors évidente : elle aide à communiquer sur un monde complexe et s'impose comme une évidence (Larson, 2011). Mais elle a aussi une autre fonction, plus cognitive. Dans un ouvrage sur l'utilisation de techniques narratives en sciences, et plus particulièrement en géographie, Vincent Berdoulay montre que « tout un arsenal de techniques langagières, toute une rhétorique, sont mis à contribution pour construire un [discours scientifique] qui vise à convaincre » (Berdoulay, 1988, p. 9). Parmi ces techniques, la métaphore a, pour V. Berdoulay, un statut particulier :

[La métaphore] est reélaboration de l'information dont l'homme dispose. Elle n'est donc pas seulement un aspect littéraire ou expressif, mais elle a aussi une valeur cognitive. Si elle s'adresse à la sensibilité - et c'est ce qui fait sa force - elle est en même temps innovation conceptuelle, création de sens. C'est pourquoi on lui reconnaît de plus en plus un rôle central et fondamental dans l'activité scientifique. La métaphore se révèle comme le procédé privilégié employé par la pensée pour innover. Elle permet au scientifique d'exprimer ses intuitions ; elle guide ses premiers pas vers la reconceptualisation des phénomènes étudiés. Elle anime de nouveaux discours. L'important n'est pas de fixer rationnellement une analogie. Au contraire, il s'agit plutôt de garder celle-ci dans le flou pour que l'incompatibilité ressentie, l'écart perçu, soient surmontés par un effort de l'imagination. Grâce au procédé métaphorique, le discours déstabilise la perception habituelle des choses et induit une redescription de la réalité, une réorganisation de l'information disponible, un mode nouveau d'appréhension des phénomènes (Ibid., p. 25).

La métaphore qui fonde la notion de services écosystémiques sert à la fois à communiquer, mais aussi à penser différemment le monde. 
Le durcissement de la métaphore, son passage au statut de notion ou de concept, est plus complexe. Des choix sémantiques sous-tendent l'expression « services écosystémiques ": là où d'autres métaphores auraient été possibles, c'est celle des services qui a été préférée. Or la notion de service en économie est très connotée : elle implique qu'il y ait un bénéficiaire de services, un fournisseur de services et un marché où faire se rencontrer fournisseur et bénéficiaire. De fait, la notion de services écosystémiques est fortement sous-tendue par l'économie.

Ainsi, lorsque Robert Costanza et ses coauteurs (Costanza et al., 1997) définissent, dans l'article le plus cité sur les services écosystémiques, cette notion, ils lient fortement la définition des services écosystémiques à leur prise en compte dans des marchés :

Les biens (comme la nourriture) et les services (comme l'assimilation des déchets) écosystémiques représentent les bénéfices que les hommes tirent, directement ou indirectement, des fonctions des écosystèmes. [...] Comme les services écosystémiques ne sont pas entièrement pris en compte dans des marchés commerciaux ou quantifiés de manière adéquate et comparable avec les services économiques et les capitaux naturels, il leur est souvent donné un trop faible poids dans les décisions politiques (Ibid., p. 253).

Si cette dimension économique était attendue de la part d'économistes, elle l'est moins de la part d'écologues. On la retrouve pourtant dans l'autre grande publication fondatrice de la notion de services écosystémiques proposée par Gretchen Daily (1997) :

Les sociétés humaines tirent nombre de biens essentiels pour leur vie des écosystèmes naturels, en particulier la nourriture tirée des mers et des animaux, les cultures, l'énergie, le bois d'œuvre et les produits pharmaceutiques. Ces biens représentent des parts importantes de l'économie. Or ces écosystèmes fournissent aussi des services de support de la vie sans lesquels nos civilisations cesseraient de prospérer. Ces services incluent la purification de l'air et de l'eau, la détoxification et la décomposition des déchets, la régulation du climat, la régénération de la fertilité des sols et la production et l'entretien de la biodiversité, lesquels sont des ingrédients clefs de nos productions agricoles, pharmaceutiques et industrielles. Ces faisceaux de services sont générés par l'interaction complexe entre des cycles naturels qui tirent leur énergie de soleil et qui opèrent à différentes échelles spatiales et temporelles (Ibid., p. 1).

L'expression " services écosystémiques » passe de la sorte du statut de métaphore à celui de concept dans un système de pensée plus large. Ce système de pensée se réfère à l'économie, ce qui était entendu dans le choix de l'image des services. L'idée d'un recours à cette sphère est de donner de la valeur à la nature pour éviter qu'elle ne soit la parente pauvre de décisions économiquement fondées : si la destruction de la 
nature n'a pas de coût pour celui qui la décide et la réalise, ce dernier ne sera pas tenté de limiter son impact sur la nature. Or si le bénéfice de la destruction d'un écosystème est individuel, son coût est collectif - le bien-être humain étant en jeu. D'où l'idée d'estimer les bénéfices rendus par la nature à l'homme pour imputer les coûts de sa destruction.

Outre son caractère métaphorique, une autre caractéristique de la notion de services écosystémiques est son caractère d'objet frontière, c'est-à-dire de concept dont la traduction du monde scientifique dont il est originaire au monde des gestionnaires a été assurée par les scientifiques eux-mêmes (Callon, 1986 ; Akrich et al., 1988 ; Star et Griesemer, 1989). Les organisations intergouvernementales (OIG) et non gouvernementales (ONG) sont les premières à s'être emparées de la notion de services écosystémiques, adoptant une notion venue du monde scientifique. Ce genre de traduction n'est pas rare dans l'histoire des sciences : le concept d'écosystème et la notion de biodiversité ont connu exactement le même processus (Castro-Larrañaga, 2013). Toutefois, ce qui est nouveau avec la notion de services écosystémiques, c'est que la traduction a été faite, au sein du MEA, directement en lien avec les acteurs clefs de la gouvernance environnementale globale (en particulier les Nations unies et la Banque mondiale) - ce qui lui assure un succès inédit.

Enfin, la notion de services écosystémiques traite des liens de dépendance que les hommes entretiennent avec leurs milieux naturels - ce qui, dans l'histoire de la modernité, est assez singulier. En effet, la modernité est caractérisée, selon Bruno Latour (1994), par le double mouvement contradictoire d'une part de mélange entre genres d'êtres entièrement nouveaux (de nature et culture à la fois), d'autre part de séparation ontologique entre nature et culture. Chacun de ces mouvements est nécessaire pour assurer l'efficacité de l'autre. Or les services écosystémiques rompent avec cette dynamique en ceci qu'ils insistent sur la dépendance de l'homme aux milieux.

Ces trois caractéristiques (caractère métaphorique, objet frontière et insertion dans la modernité) génèrent une tension fondamentale à l'usage de l'expression "services écosystémiques », qui se retrouve dans les débats scientifiques entourant la notion.

\section{Débats et enjeux autour de la notion de services écosystémiques}

L'apparente neutralité des services écosystémiques ne tient pas longtemps quand on regarde la manière dont la notion est débattue dans la littérature. Il serait vain de tenter d'être exhaustif quant à ces débats compte tenu de l'intense production bibliographique qui entoure les services écosystémiques (figure 2). Cependant, un rapide survol des 
positionnements à l'intérieur de ces débats permet de se rendre compte des enjeux de cette notion.

On peut schématiquement distinguer deux types de littérature. Une partie de la littérature tient la notion de services écosystémiques pour acquise et s'interroge sur la manière de la traiter scientifiquement ou de la mettre en politique. Une autre approche, au contraire, propose une critique affirmée de la notion de services écosystémiques. Bien évidemment, cette distinction a un caractère artificiel : des articles classés dans l'une ou l'autre de ces approches peuvent avoir un positionnement plus nuancé. Cette tendance s'est affirmée dans la bibliographie ces dernières années, au point qu'un troisième courant, attentif aux dangers potentiels de la notion, s'affirme.

La volonté d'opérationnaliser la notion de services écosystémiques est le titre même d'un article que Gretchen Daily et Pamela Matson (2008) donnent à un article programmatique. Elles proposent trois pistes pour opérationnaliser la notion : il faut, selon elles, développer la connaissance scientifique et la cartographie des fonctions produites par les écosystèmes et assurant des services ; créer des institutions financières, politiques et de gouvernance des services; et trouver le moyen de mettre en œuvre ces politiques dans des contextes précis. Chacune de ces pistes renvoie à une sphère particulière : la première renvoie à la réalité écologique que cette notion prétend décrire ; la sphère institutionnelle renvoie aux institutions à créer pour gérer le rapprochement entre écologie et économie que la notion de services écosystémique implique ; la troisième dimension renvoie aux politiques qui peuvent mettre en œuvre cette notion. Ces trois dimensions sont aussi celles qui sont présentes dans la plupart des articles, tant ceux qui cherchent à opérationnaliser les services écosystémiques que ceux qui en proposent une critique.

D'un point de vue écologique, l'opérationnalisation des services écosystémiques passe, par exemple, par la volonté de préciser la capacité de stockage de carbone d'une forêt, la purification de l'eau dans un périmètre d'eau potable ou la dégradation des sols. Ainsi va-t-on trouver tout un ensemble d'articles qui cherchent à caractériser le potentiel de services écosystémiques d'une région, ou d'un type d'écosystème. Ces approches ont des visées scientifiques, mais aussi, on s'en doute, opérationnelles : quantifier les services écosystémiques est indispensable pour décider où mettre en place des politiques de protection de ces services. Un article que Robin Naidoo signe avec plusieurs coauteurs (2008) propose une cartographie des zones de production de services écosystémiques afin de mieux cibler des écorégions où la protection de services écosystémiques et de biodiversité pourrait être maximisée.

Le passage d'institutions aptes à gérer les services écosystémiques se fait souvent par le truchement d'une évaluation économique des services 
écosystémiques. Ainsi, à la suite du MEA, un groupe de recherche composé d'économistes s'interrogeant sur les différentes manières de donner de la valeur aux services écosystémiques et à la biodiversité s'est mis en place : il s'agit du TEEB (initiative pour l'économie des services écosystémiques et de la biodiversité) (Sukhdev, 2010). Ces différentes manières d'évaluer les services écosystémiques se retrouvent dans des mécanismes de marché des services écosystémiques (Broughton et Pirard, 2011).

Mais le marché n'est qu'une des institutions possibles. Ainsi la définition de valeurs économiques à la nature n'a-t-elle pas comme unique but de faire entrer celle-ci dans le marché. Dans un article qui fait le point sur les différentes méthodes d'évaluation économique des services écosystémiques, Jean-Michel Salles (2011) précise que l'utilité d'une telle démarche " n'est pas de donner une valeur économique à la nature, ce qui est inutile, mais de traduire la valeur des pertes consécutives à la destruction des écosystèmes en des termes qui permettent de comparer les services écosystémiques avec d'autres enjeux sociétaux » (p. 478). Il identifie quatre principaux enjeux autour de la création de valeurs des services écosystémiques : maximiser l'efficacité dans les politiques de préservation, évaluer un potentiel de services, fournir des outils d'aides à la décision et poser la question des services pour permettre la création de prérequis dans la prise de décision.

L'estimation économique de la valeur des services écosystémiques est de ce fait en fort lien avec les politiques publiques. Cela renvoie à la troisième piste proposée par G. Daily et P. Matson pour l'opérationnalisation des services écosystémiques : la mise en place de politiques précises capables d'assurer leur utilisation sur le terrain. Nombre d'articles mettent l'accent sur le fait que la dégradation des services environnementaux n'est pas que due au fait qu'ils ne soient pas pris en charge par le marché. C'est le cas plus particulièrement d'un article dont le titre, La tragédie des services écosystémiques (Lant et al., 2008) fait écho à une publication demeurée célèbre dans l'appréhension des problèmes environnementaux (Hardin, 1968). Les auteurs de cet article montrent que des causes sociales complexes (complex social trap) sont à l'origine de la « tragédie des services écosystémiques » : les incitations économiques encouragent la dégradation des services publics au bénéfice de la production de biens privés, les droits de propriété renforcent les défaillances du marché, il n'existe pas d'échelles de gestion adéquate pour ces services, etc. Aussi suggèrent-ils la création d'institutions chargées de prendre en compte la dégradation des services écosystémiques à des échelles plus efficaces.

Le statut à donner à ces trois pistes d'opérationnalisation des services écosystémiques diffère cependant selon les auteurs. En effet, l'opérationnalisation des services écosystémiques pose de nombreux 
problèmes - au point que certains auteurs doutent de la possibilité, voire de l'opportunité, de l'utilisation de la notion de services écosystémiques. Ces critiques des services écosystémiques peuvent être différenciées entre des critiques d'ordre conceptuels, dont le but est d'en améliorer l'efficacité, et des critiques plus fondamentales portant sur les dimensions éthiques sous-tendues par la notion de services.

Nombre d'auteurs critiquent le soubassement écologique de la notion de services écosystémiques. Émanant principalement de l'écologie, ces auteurs mettent l'accent sur le fait que les services écosystémiques, tels qu'ils ont été définis dans le MEA, confondent ce qui relève de la structure des écosystèmes, de leurs fonctions et de l'utilisation de ces fonctions par l'homme. Ainsi les services de supports renvoient-ils plus à des processus écosystémiques qu'à des fonctions d'écosystèmes. La photosynthèse ou les cycles de l'eau existent indépendamment de l'utilisation qui en est faite par l'homme : ils sont produits en tant que tels. De la confusion entre processus, fonctions et services résulte un flou dans la notion de services écosystémique dont l'appréhension ne fait pas l'unanimité (Barnaud et al., 2011). De même, nombre de services manquent à l'appel, d'autres appartiennent à plusieurs catégories à la fois, etc.

Pour R. Costanza (2008), ce flou peut être interprété comme une caractéristique même de la notion. Il appelle à en avoir une vision pragmatique :

Les services écosystémiques sont définis comme "les bénéfices que les hommes tirent des écosystèmes " (Millennium Ecosystem Assessment (Program), 2005a). Je pense que c'est une bonne définition, car elle suffisamment large et vague. La définition inclut à la fois les bénéfices que l'homme perçoit, et ceux qu'il ne perçoit pas. [...] Les écosystèmes sont des systèmes complexes, dynamiques et adaptatifs, avec des rétroeffets non linéaires, des effets de seuil, etc. [...]. Nos systèmes de classification et de définition des services écosystémiques doivent partir de cette base (Costanza, 2008, p. 351-352).

Toutefois, d'autres auteurs insistent sur le fait que ce flou devient un problème pour la science. Pénélope Lamarque, Fabien Quétier et Sandra Lavorel (2011) montrent que si le flou peut avoir une utilité pédagogique et politique, il se traduit par une diversité de définitions des services écosystémiques qui recoupe une diversité de visions des milieux et de leur gestion (entre approches anthropocentrées et biocentrées par exemple). Or ces auteurs montrent, dans le cas des pâturages, que cette diversité pose problème quand on cherche à mesurer précisément des services écosystémiques dans des milieux, car elle aboutit à des évaluations très différentes des services qui y sont fournis. Pour eux, il est nécessaire de s'entendre sur des définitions précises des services écosystémiques pour permettre des évaluations qui fassent minimalement consensus. 
D'autres auteurs vont plus loin, et en viennent à rejeter la notion elle-même. Pour Richard Norgaard (2010), autant la métaphore de service écosystémique a pu avoir une utilité pour aider à comprendre la dépendance de l'homme des écosystèmes, autant sa domination comme unique métaphore pour appréhender les rapports hommes/milieux cache la diversité de ces rapports et fait courir le risque de fermer plus de portes qu'elle n'en ouvre. Cet auteur s'inscrit dans la lignée de travaux qui critiquent la vision non seulement anthropocentrée des services écosystémiques, mais aussi occidentalo-centrée, voire profondément capitaliste (voir en particulier l'ouvrage de Virginie Maris, 2014).

On peut dans la même veine trouver un ensemble de travaux qui montrent que si la notion de services écosystémiques est intéressante en tant que notion métaphorique, son utilisation dans le contexte néolibéral actuel ouvre la porte à une mise sur le marché de la nature qui peut avoir des effets contre-productifs sur le long terme (Gomez-Baggethun et Ruiz, 2011). En effet, pour ces auteurs si le capitalisme est la cause de la dégradation des services écosystémiques, vouloir lutter contre cette dégradation par une mise sur le marché de la nature revient à résoudre le problème par la cause même du problème. Une initiative, Nature $\mathbb{C}$, a notamment vu le jour au début des années 2010 pour dénoncer la mise sur le marché de la nature. L'introduction du numéro spécial de la revue Development and change qui est tiré du colloque Nature (C) commence ainsi :

《 La nature est morte. Longue vie à la nature (C) ! " Ce cri de ralliement inspire (non officiellement) nombre de politiques environnementales contemporaines. Il est argumenté que des méthodes nouvelles et innovantes sont nécessaires pour répondre aux nombreux problèmes environnementaux auxquels l'homme est confronté, que les marchés capitalistes sont le moyen idéal de porter ces politiques. En effet, les forces du marché ont trouvé leur place dans les politiques de conservation et les politiques environnementales à un niveau qui aurait paru encore inimaginable il y a de cela vingt ans seulement. Les paiements pour services environnementaux, les REDD et les marchés du carbone, la mise sur le marché de la conservation, les banques d'espèces $[\ldots]$ ne sont que quelques-uns des mécanismes de marché qui ont gagné en popularité au cours des dernières années, en dépit - ou peut-être à cause de - de la crise financière actuelle (Arsel et Büscher, 2012).

Ces critiques radicales irriguent un grand nombre de penseurs, en particulier dans les sciences sociales. Par ailleurs, des doutes émergent quant à la possibilité même d'opérationnaliser la notion de services écosystémiques dans des politiques publiques. Ces doutes ne portent pas tant sur la notion elle-même, dont l'efficacité scientifique semble pour ces auteurs démontrée, mais sur sa possibilité d'utilisation dans 
le cadre de politiques. C'est le cas des travaux d'Alain Karsenty sur les forêts tropicales. Dans un premier article écrit en 2007 avec Romain Pirard, il doutait de l'efficacité des mécanismes de rémunération des déforestations évités et plaidait plutôt pour renforcer les dispositifs existants et supprimer les incitations perverses à la déforestation (Karsenty et Pirard, 2007). Dans un autre article écrit avec Symphorien Ongolo, A. Karsenty s'interrogeait sur les présupposés mêmes de ces mécanismes, qui tiennent pour acquis le fait que les États sont des acteurs rationnels qui prennent des décisions en fonction de leurs intérêts (selon le présupposé de l'homo economicus) : or c'est loin d'être le cas de nombre d'États dits fragiles, dont les mécanismes de prise de décision ne peuvent pas être considérés comme rationnels (Karsenty et Ongolo, 2012).

L'appropriation par les scientifiques de la notion de services écosystémiques fait largement débat - au point qu'il est difficile de parler au singulier de cette notion. Pourtant, il nous semble que se détache, dans la littérature scientifique parue ces dernières années, une tendance à traiter cette notion avec prudence, mais en reconnaissant l'intérêt qu'elle peut avoir pour un certain nombre de champs. Nombre d'articles récents proposent une approche plus nuancée de la notion de services écosystémiques. C'est le cas, par exemple, d'un article écrit par Jessica Dempsey et Morgan Robertson (2012), qui montre trois choses : tout d'abord, qu'en dépit des nombreuses craintes relatives à la marchandisation de la nature qui ont émergé quant aux services écosystémiques, peu d'évidences qu'un tel phénomène s'est réellement mis en place apparaissent. Au contraire, les politiques observées s'éloignent considérablement de la doctrine néolibérale tant redoutée. Ensuite, ces auteurs montrent que le monde des services écosystémiques est lui-même traversé de tensions très fortes entre différentes acceptions du terme. Enfin, ces auteurs montrent que ces tensions ouvrent de très larges opportunités pour l'appréhension des débats sur les services écosystémiques.

C'est dans cette acception assez pragmatique de la notion de services écosystémiques que nous situons notre approche dans cet ouvrage. Notre objectif est d'identifier les opportunités ouvertes par cette notion, mais aussi les portes qu'elle ferme et les dangers qu'elle recèle. Ce double souci va nous animer tout au long de cet ouvrage. Mais cette approche doit être construite scientifiquement pour qu'elle ne confine pas à un vague pragmatisme.

\section{Political ecology des services écosystémiques}

Ces éléments imposent de mettre en question le caractère universel de la notion de services écosystémiques : son évidence n'est qu'apparente. Pour nous, fonder une approche raisonnablement critique de cette notion 
passe par la compréhension des enjeux qui se déroulent dans quatre sphères dans lesquelles elle se déploie : la sphère écologique, la sphère économique, la sphère politique et la sphère sociale.

Pour aborder ces sphères, nous avons fait le choix d'une approche dite de Political ecology. La Political ecology est un courant scientifique, principalement anglo-saxon, dont le champ d'études se situe à la croisée entre l'écologie, les politiques environnementales et les populations qui transforment les milieux et sont l'objet des politiques. Interdisciplinaire par la nature, la Political ecology se caractérise à la fois par une approche critique de la manière de formuler les problèmes environnementaux et par une volonté de dépasser la critique pour mettre en œuvre des outils d'analyse et de résolution de ces problèmes.

La Political ecology est de ce fait à la fois critique, compréhensive, et force de proposition. Elle est critique en ce qu'elle considère que les discours sur l'environnement, y compris les discours scientifiques, ne sont pas neutres. Ils traduisent non seulement des visions du monde, mais aussi les intérêts de leurs locuteurs. Or ces discours sont performatifs dans le sens où ils rendent possible une action sur la nature. Mais la Political ecology est compréhensive en ceci qu'elle considère que les discours sont triplement contraints : ils reposent sur une réalité, celle de l'environnement dont ils qualifient l'état; ils dépendent des outils à la disposition des scientifiques pour rendre compte de cette réalité ; et ils traduisent enfin les préoccupations du contexte dans lequel ils sont émis. Enfin, la Political ecology cherche à proposer des approches alternatives des problèmes environnementaux qu'elle étudie : en reconnaissant à la fois la réalité de ces problèmes et la manière dont ils sont construits, elle ouvre la porte à des approches alternatives de ces problèmes - et donc des politiques appelées à les gérer.

On comprend, compte tenu de ce que nous avons dit des services écosystémiques, l'intérêt d'une telle approche pour étudier cette notion : signe d'un renouveau des politiques environnementales, elle pose au moins autant de problèmes qu'elle n'en résout. Elle appelle une approche critique, mais est suffisamment intéressante pour mériter d'être étudiée en détail. Scientifiquement, la notion de services écosystémiques invite à une interdisciplinarité large, qui irait de la manière dont sont construits les objets scientifiques à leur sens pour des acteurs de terrain et leur opérationnalisation concrète. La Political ecology fournit un cadre à cette étude.

Ayant opté pour une telle approche, nous faisons bien entendu le choix de considérer la sphère politique comme primordiale. La notion de services écosystémiques est fortement marquée par les réseaux scientifiques qui l'ont portée, par le contexte qui l'a vue naître et s'affirmer 
et par les acteurs qui l'utilisent. Dès lors, il nous apparaît nécessaire de qualifier les enjeux et les limites de cette notion en fonction des acteurs qui ont participé à sa formulation et en fonction des échelles auxquelles ces acteurs parlent. Qui parle de services écosystémiques ? D'où viennent ces locuteurs ? Quels débats entretiennent-ils ? Quelles voix se font-elles le plus entendre? Lesquelles perdent en audience?

Ce discours n'est bien entendu pas détachable de la réalité dont il rend compte ni du contexte dans lequel il est émis. En ceci, il ne saurait être considéré comme un simple discours. En termes contextuels, par rapport à la période que nous vivons, de quoi ces discours sont-ils le signe ? Quelles transformations des rapports hommes/milieux, et plus particulièrement des rapports capitalisme/milieux et de la gouvernance environnementale, la notion de services écosystémiques dessine-t-elle ? En termes d'épistémologie des sciences et de la modernité (en particulier dans ses rapports à l'économie), la notion de services écosystémiques est le signe d'inflexions qu'il importe, par rapport aux acteurs et aux enjeux identifiés ci-dessus, de qualifier. Enfin, ce discours est censé qualifier une réalité. Quelles modifications dans la description de la réalité la notion de services écosystémiques engendre-t-elle ? Quelles portes sont-elles ouvertes et fermées ? Comment cette notion fonctionne-t-elle concrètement? Quelles modifications dans l'appréhension de la réalité ce fonctionnement implique-t-il ?

Cet ouvrage est fondé sur la rencontre entre chacune des trois sphères dans lesquelles se déploie la notion de services écosystémiques. La première partie du livre cherchera à en circonscrire le périmètre de validité, les potentialités et les directions qu'elle prend. Définir un périmètre de validité a pour bu de remettre en question l'universalité de cette notion en qualifiant les lieux (c'est-à-dire les rencontres entre acteurs et échelles) où elle est pertinente. Après avoir posé les bases théoriques et méthodologiques de l'analyse dans le chapitre 1 , le chapitre 2 cherchera à circonscrire le périmètre de validité de la notion de services écosystémiques par une approche généalogique : cela nous permettra de mieux la qualifier et d'en cerner les enjeux, en particulier épistémologiques et sociaux. En se consacrant à l'actualité récente des services écosystémiques, le troisième chapitre analysera la manière dont les déploiements de la notion activent un certain nombre de potentialités des services écosystémiques. Nous verrons alors que si l'économicisation de la nature incluse dans la notion de services écosystémiques ne semble pas d'actualité, il n'en demeure pas moins qu'elle est au cœur de la refondation de la gouvernance environnementale.

La seconde partie analysera plus concrètement des contextes dans lesquels cette notion est utilisée, en se demandant comment elle est 
concrètement appropriée par des acteurs politiques. Trois exemples seront étudiés, tous tirés des contextes de forêts tropicales dans lesquels cette notion est supposée se déployer avec une vigueur particulière. On commencera par une étude des projets REDD, des réseaux qui les portent et, surtout, de ceux qui les contestent (chapitre 4). On retrouvera, en analysant le mode de fonctionnement de ces réseaux, certaines des caractéristiques de la notion de services écosystémiques. Ensuite, nous approfondirons deux contextes nationaux celui du Gabon (chapitre 5) et celui du Brésil (chapitre 6). Nous montrerons que, dans ces pays, la notion de services écosystémiques a peu modifié les politiques concrètes dans lesquelles elle s'est plutôt insérée comme un outil supplémentaire à la disposition d'acteurs politiques pour renforcer des politiques qu'ils menaient déjà.

Notre troisième partie montrera que, en prétendant traiter les rapports hommes/milieux en une seule notion, une notion de compromis, les services écosystémiques, bien compris, offrent tout de même un cadre d'analyse pertinent dans certains usages bien définis. La dernière partie de notre ouvrage sera consacrée à ce que la mesure (chapitre 7) et la représentation spatiale (chapitre 8 et 9), bien comprises, de services écosystémiques permet d'ouvrir comme pistes d'actions - là encore dans le cadre de forêts tropicales.

En utilisant une approche de Political ecology pour aborder la notion de services écosystémiques, nous chercherons non seulement à mettre la notion de services écosystémiques à l'épreuve d'une approche scientifique particulière, mais aussi à tester, pour un public francophone au moins, la performativité de la Political ecology. Approche scientifique peu connue en France où les sciences restent structurées autour de disciplines ou de sous-disciplines, la Political ecology fait l'objet, ces dernières années, d'un intérêt certain. Apparue dans les publications de langue française à la fin des années 2000, en particulier dans la revue Natures Sciences Sociétés (Benjaminsen et Svarstad, 2009 ; Castro-Larrañaga, 2009), elle a donné lieu à un ouvrage collectif qui en circonscrit le champ et en présente les multiples facettes à un public francophone (Gautier et Benjaminsen, 2012). Mais à notre connaissance, cet ouvrage est le premier en langue française qui met à l'épreuve la Political ecology d'un objet particulier en l'occurrence la notion de services écosystémiques. 


\section{Première Partie}

À LA RECHERCHE DU PÉRIMÈTRE DE VALIDITÉ DES SERVICES ÉCOSYSTÉMIQUES 


\section{Introduction à la première partie}

Cette partie vise à circonscrire le périmètre de validité de la notion de services écosystémiques en retraçant son origine et en suivant son déploiement. Pour ce faire, le premier chapitre propose un cadre théorique et historique qui permet de considérer cette notion comme un dispositif au sens foucaldien du terme. Apparue à un moment donné de l'histoire de l'Occident, en plein débat sur la légitimité et la nature de la modernité écologique, la notion de services écosystémiques a été créée pour intervenir dans ces débats afin de justifier ou infléchir les politiques environnementales. D'où l'importance de revenir sur le contexte de sa création, sur les courants scientifiques qui l'ont portée : en faisant, une généalogie de la notion de services écosystémiques, le deuxième chapitre analyse les raisons pour lesquelles cette notion a été créée, les milieux dans lesquels elle fonctionne, les débats auxquels elle participe. Nous verrons que le monde de la conservation, ses justifications et ses modes de financement sont très clairement au cœur de l'histoire de la notion de services écosystémiques.

Mais un dispositif est très évolutif : créé à un moment donné pour répondre à une fonction précise, il évolue en fonction du contexte et prend son sens au fur et à mesure de son déploiement. Le deuxième et surtout le troisième chapitre décrivent ces évolutions et les sens que prend cette notion au fur et à mesure de son utilisation dans différentes sphères sociales, scientifiques et politiques, permettant ainsi de proposer une vision synthétique des sens qu'elle prend aujourd'hui. 


\section{DeUXIÈMe PaRTIE}

QUAND LES SERVICES ÉCOSYSTÉMIQUES DEVIENNENT DES POLITIQUES 


\section{Introduction à la deuxième partie}

Le destin de la notion de services écosystémiques est assez ambivalent. Construite comme outil de légitimation des politiques de conservation, elle permet de renouveler le vocabulaire des acteurs de la conservation tout en restructurant ou légitimant certaines communautés scientifiques. Elle a cependant du mal à percoler en dehors de ces mondes, comme l'a montré la conférence de Rio, où cette notion est finalement peu utilisée. En outre, elle perd de son homogénéité conceptuelle au fur et à mesure de son utilisation, et se diffracte entre de multiples sens (figure 12).

L'objectif de cette deuxième partie est d'analyser ce qui se passe lorsque la notion de services écosystémique devient un concept opératoire intervenant dans le monde du développement ou des politiques publiques. Pour homogénéiser les cas d'études, nous avons centré nos travaux sur les contextes forestiers tropicaux. Trois cas seront exposés dans cette partie, faisant chacun l'objet d'un chapitre : le premier exemple analyse les réseaux promouvant ou s'opposant aux REDD. Les oppositions aux REDD nous ont semblé particulièrement intéressantes à étudier en ceci qu'elles traduisent les difficultés de mise en œuvre de ces outils dans des situations concrètes, et plus encore la grande défiance dont ils font l'objet. Un grand nombre d'acteurs de la société civile voient dans ces outils la main du capitalisme, et le rejettent violemment en s'opposant au principe de marchandisation de la nature.

Ce n'est pourtant pas ce qui semble effectivement se passer lorsque l'on se confronte à la réalité de l'utilisation de ces outils dans des contextes nationaux. Cette réalité, nous nous y sommes confronté en étudiant l'utilisation des services dans deux pays de deux grands massifs forestiers tropicaux, le Gabon dans le bassin du Congo et le Brésil en Amazonie. Dans les deux cas, des politiques de paiements de services environnementaux sont explicitement invoquées. Qu'ont-elles changé aux pratiques de gestion des milieux naturels déjà existantes? Les deux cas d'études montrent que ce sont surtout des repositionnements de politiques déjà existantes que l'on observe, le poids des contextes nationaux étant très important pour comprendre la manière dont est appliquée cette notion. Plus que des outils de " marchandisation de la nature ", elles servent plutôt à renforcer les rhétoriques et les positionnements des acteurs qu'à infléchir leurs logiques d'action.

Cette place plus importante que prend l'État dans la définition des politiques environnementales va finalement dans le même sens que celui 
que nous avons évoqué dans le chapitre 3 en parlant de K. Polanyi : loin d'être un outil de marchandisation de la nature, la notion de services écosystémiques pourrait être un moyen de revenir sur certains excès du capitalisme. Mais cette contestation est fortement limitée. Que ce soit les projets REDD ou dans les politiques de PSE au Brésil ou au Gabon, on est surtout frappé par le faible nombre de projets existants. Pour l'instant, ces projets ont faiblement percolé dans le monde de la gestion de l'environnement, même si leur importance va croissant. 


\section{Troisième PARTIE}

ENJEUX DE LA QUANTIFICATION ET DE LA SPATIALISATION DES SERVICES ÉCOSYSTÉMIQUES 


\section{Introduction à la troisième partie}

L'ambition de cet ouvrage est d'analyser, de façon fine et critique, les limites du domaine de validité de la notion de services écosystémiques. Cette analyse, guidée par une démarche de type Political ecology, a été développée dans les parties précédentes selon différents points de vue : la généalogie de la notion et son utilisation dans différentes arènes politiques. De fait, il a été montré que cette notion renvoie à plusieurs catégories de discours (politique, sociale, gestionnaire, heuristique, sécuritaire, économique) qui relèvent schématiquement de deux dimensions : une dimension pédagogique globale ou conceptuelle (faire prendre conscience de la dépendance des sociétés aux objets de nature) et une dimension opérationnelle (améliorer les pratiques de gestion et de conservation). De fait, la dimension pédagogique globale est en partie à l'origine de la mise à l'agenda politique de la notion de services écosystémiques, mais cette dernière recouvre des réalités multiples. Certains auteurs et certaines institutions pensent qu'elle offre également un cadre efficace pour l'élaboration et/ou à la mise en œuvre de politiques publiques d'amélioration de la gestion et de la préservation des milieux naturels. Or, si la dimension pédagogique globale semble bien établie depuis la publication du Millenium Ecosystem Assessment (MEA) en 2005, la dimension opérationnelle fait l'objet de nombreux débats. Ces débats ont structuré l'évolution de la notion après la publication du MEA, se sont retrouvés au cours de la conférence de Rio et ont été confirmés par l'étude de contextes spécifiques au Brésil ou au Gabon : in situ, le caractère opérationnel des services écosystémiques est discutable.

L'objectif de cette partie est d'analyser un volet particulier du dispositif environnemental que représentent les services écosystémiques, celui des instruments de quantification et de représentation qu'elle mobilise. De fait, G. Daily et S. Matson (2008) identifient la nécessité de construire un champ disciplinaire visant à quantifier (souvent au travers d'indicateurs) et à cartographier les services écosystémiques comme un des trois besoins prioritaires afin d'opérationnaliser la notion de services écosystémiques. De même, cette nécessité est reconnue par l'Union européenne comme indispensable afin d'atteindre les objectifs fixés par la stratégie européenne pour la biodiversité. Or, la production d'indicateurs et la spatialisation de services écosystémiques (ou de processus sous-jacents aux services) ne vont nécessairement pas de soi ; elles requièrent la mobilisation de connaissances, de compétences, de méthodologies et d'acteurs. Cette 
partie est fondée sur l'idée qu'analyser ces pratiques revient à porter un regard critique sur l'ensemble du dispositif au sein duquel elles s'insèrent ou de la politique à laquelle elles concourent. Ainsi, nous montrerons que l'examen des conditions particulières de mise en œuvre de la mesure et de la représentation de la notion de services écosystémiques permet de mieux en cerner le domaine de validité et d'usage.

Concrètement, cette partie est organisée autour de trois chapitres. Premièrement, nous rappellerons que, derrière la notion de services écosystémiques, il existe une large gamme de processus biophysiques dont la complexité intrinsèque limite fortement notre capacité à proposer des mesures simples et précises de services écosystémiques. Nous montrerons également que la caractérisation de la structure et de la dynamique des paysages est potentiellement une approche susceptible de contourner cette limitation au travers d'une réflexion plus générale sur les compromis et les synergies entre services écosystémiques. Deuxièmement, nous analyserons les choix méthodologiques et les enjeux techniques liés à la spatialisation des services écosystémiques afin de démontrer la difficulté de les représenter spatialement. Si les deux premiers chapitres de cette partie reposent exclusivement sur l'analyse de la littérature scientifique, nous revenons, dans le troisième, sur l'arène politico-scientifique que constitue le MEA. En effet, après avoir illustré la complexité des processus de quantification et de spatialisation des services écosystémiques, nous analyserons comment ces outils (et plus particulièrement les cartes) sont mis en avant, utilisés et justifiés. Cette analyse soulignera notamment la faible place qui est faite aux dimensions critiques et politiques lors de la mobilisation de ces outils et nous conduira à conclure provisoirement que, d'un point de vue opérationnel, les services écosystémiques sont clairement un outil de sensibilisation globale, potentiellement un instrument de débat, mais probablement pas un outil d'évaluation ni de prise de décision qui reste in fine politique. 


\section{Conclusion}

L'objet de ce livre était de traiter de manière critique et constructive les services écosystémiques en employant une démarche de Political ecology. Nous avons voulu avoir une approche intégrée de cette notion, en retraçant son histoire, en regardant ses usages et en analysant son utilité pour la science et la gestion environnementale. Ce faisant, nous avons progressivement délimité son périmètre de validité. C'est sur ce périmètre que nous voudrions revenir au moment de conclure cet ouvrage.

La notion de services écosystémiques vient du monde de la conservation. Elle a été mise en place pour renforcer les argumentaires des conservationnistes en insistant sur le fait que protéger la nature, c'était protéger le bien-être humain ; elle place la nature au service de l'homme et recourt à une métaphore d'origine économique pour appeler à prendre en compte dans les mécanismes de prise de décision les pertes engendrées par la destruction ou la pollution d'écosystèmes naturels. Cela se comprend dans le cadre d'une modernité écologique fortement contestée : la notion de services écosystémiques permet de relégitimer la protection de l'environnement, et de considérer la nature non plus comme opposée au développement, mais comme étant condition du bien-être humain. Les usages qui en ont été faits à la Conférence de Rio+20 (figure 12) en montrent les différentes applications, principalement rhétoriques. La notion de services écosystémiques sert d'abord à fonder un argumentaire économique et social, en termes de richesse ou de bien-être. Elle sert ensuite des fins politiques (apaiser des conflits), heuristiques (analyser différemment les anthroposystèmes) et gestionnaires (gérer les milieux).

Ses présupposés sont bien évidemment contestables; le bien-être est bien plus large que ce qu'en dit le MEA, la nature n'est pas au service de l'homme, sa marchandisation est un risque non négligeable. Mais cette notion est d'abord une notion de combat. Combat interne à la modernité écologique, et combat interne à la science. Le MEA, qui a intronisé les services écosystémiques, cherchait en les promouvant autant à construire un cadre rhétorique pour la justification de la protection de la biodiversité qu'à promouvoir le concept phare de certains courants de la biologie de la conservation. De ce fait, la notion de services écosystémiques apparait comme un dispositif de la modernité écologique où savoirs et pouvoirs s'allient pour construire un outil de gouvernementalité. C'est un construit stratégique qui émerge au sein d'un rapport de forces donné et qui est lié à des savoirs spécifiques. 
La notion de services écosystémiques est cependant loin d'être homogène, et la façon dont elle est appliquée dépend fortement de l'histoire des acteurs qui participent à son application. Différents courants de pensée vont chacun lui donner un sens. Le troisième chapitre et la deuxième partie de l'ouvrage montrent que chaque acteur la reçoit en fonction de son histoire propre. Flairant le capitalisme, certaines ONG, celles qui se rencontrent dans les forums globaux alternatifs, y voient un instrument de marchandisation de la nature - craintes dont se fait écho une certaine partie de la communauté scientifique. Mais au Gabon comme au Brésil, c'est plutôt à une requalification des politiques publiques, qui ne changent pas dans leurs objectifs ni même dans leur fonctionnement, que l'on assiste. Les paiements pour services environnementaux fonctionnent comme une promesse performative, celle d'une nouvelle manne pour financer la conservation; que l'argent tarde à arriver, que cet outil peine pour l'instant au moins à se transformer en outil de politiques publiques, ne change rien au fait que ce nouvel habillage permet de relégitimer ou de requalifier certaines politiques. Tout se passe comme si cette rhétorique fournissait des arguments supplémentaires pour justifier des politiques et chercher de nouveaux bailleurs.

Ces usages des services écosystémiques reposent la question que nous avons abordée dans le premier chapitre de la place de cette notion par rapport aux évolutions conjointes de la modernité et de la théorie économique - question posée par Karl Polanyi en son temps. Ce que l'on observe pour l'instant, c'est que loin de marquer une marchandisation de la nature, et donc un approfondissement du capitalisme, elle sert à renforcer le pouvoir de l'Etat dans ses politiques de conservation et à augmenter le spectre des écosystèmes qui doivent être protégés - face à certains acteurs économiques toujours prompts à contester le bien-fondé de la conservation.

Pour l'instant au moins, la notion de services écosystémiques a surtout une fonction pédagogique et rhétorique. Cela est d'autant plus vrai que le processus qui a accompagné son développement a aussi permis de penser les problèmes liés à la dégradation des milieux naturels en termes de processus. Le MEA a donné aux problèmes environnementaux une vraie profondeur historique, les a replacés dans une temporalité plus large que la temporalité humaine. Si la notion de services écosystémiques peine à se transformer en outil de marchandisation de la nature, ce n'est peutêtre pas tant du fait des usages qui en sont faits que du fonctionnement même des écosystèmes. Dit autrement, le fait que la notion de services écosystémiques ne fonctionne pas encore comme un Cheval de Troie de la marchandisation de la nature n'est peut-être pas tant dû aux intentions des acteurs qu'au fait qu'elle ne peut pas servir à fonder un marché. La théorie économique nous apprend que pour fonder un marché, il faut des 
droits de propriété (pour savoir qui vend), une institution de régulation et une unité d'échange. Un acheteur a besoin de savoir ce qu'il achète de manière certaine.

Or la troisième partie de notre ouvrage sur la spatialisation des services écosystémiques montre qu'il apparaît extrêmement difficile de quantifier les services rendus par les écosystèmes. Les difficultés sont en effet considérables : les hypothèses, les choix d'experts, les échelles d'observations, les données disponibles, les simplifications que l'on doit faire pour disposer d'indicateurs de fonctions ou propriétés (plus que de processus et de services) d'accès assez facile pour comparer un grand nombre de sites et analyser des variations spatio-temporelles rendent, en l'état actuel des connaissances, la mise en place d'indicateurs stables et globaux de services totalement illusoire. Il est difficile de proposer des indicateurs d'un service à l'échelle d'un milieu, plus difficile encore de parler de compromis de services à cette échelle. La compréhension des liens entre processus physiques, indicateurs de services écosystémiques et utilisation de ces services par l'homme est un défi pour la recherche, d'autant plus que chaque service écosystémique a son propre fonctionnement. Or la géographie comme l'écologie nous rappellent que cela n'a pas de sens de parler d'un service indépendamment des processus fonctionnant sur un territoire. De ce fait, regrouper des services dans des indicateurs de compromis de services est un défi plus grand encore. Alors parler de services à une échelle plus large, à l'échelle d'une région ou d'une écorégion, est extrêmement hasardeux.

Le danger pour les scientifiques est d'oublier les limites de cet exercice, d'accorder trop de crédit et de confiance aux chiffres et aux cartes qu'ils produisent. La volonté « d'être utile » est extrêmement forte chez les scientifiques. Notre livre l'a montré, leurs interventions dans la sphère publique recoupent les convictions profondes de communautés épistémiques désireuses de défendre des causes. Cette volonté peut les amener à faire trop confiance à leurs propres outils. En matière de services écosystémiques, l'utilité de la science n'est-elle pas plus de reconnaître ses propres limites que de chercher à tout prix à proposer des outils de mesure et des indicateurs ? Certes, la notion de services écosystémiques fonctionne comme un excellent outil heuristique, qui permet de rechercher des compromis de services, de comprendre les liens entre ces compromis et différents drivers socio-économiques, etc. Elle ouvre des pans de recherche entiers.

Mais il ne faudrait pas que les scientifiques se mettent à croire à leurs propres objets, à les « essentialiser ». La science n'en fait-elle pas trop autour des services écosystémiques ? La volonté des chercheurs d'intervenir dans le débat public n'a-t-elle pas produit une bulle autour des services écosystémiques ? Cette bulle n'a-t-elle pas atteint une taille 
bien supérieure à l'utilité réelle de cette notion ? Nous le pensons, et nous espérons avec cet ouvrage avoir dégonflé un peu la bulle, en avoir limité les usages irraisonnés. C'est le sens que nous avons donné à notre démarche de Political ecology. En ayant une vision intégrée des services écosystémiques, de l'histoire des sciences aux spatialisations de services en passant par les usages politiques de cette notion, nous avons voulu en circonscrire le périmètre de validité et attirer l'attention sur ses limites et donc les risques de son usage non maîtrisé. Ce faisant, et c'est là sans doute un des paradoxes de notre livre, nous avons sans doute contribué à gonfler encore un peu plus la bulle. Mais il nous semble nécessaire de l'avoir fait. 


\section{Les auteurs}

Xavier Arnauld de Sartre est chargé de recherches au Centre national de la recherche scientifique, directeur adjoint de l'UMR Société environnement territoire (CNRS/Université de Pau et des Pays de l'Adour). Il a travaillé sur la place des populations rurales dans des territoires soumis soit à de fortes pressions productivistes (comme la Pampa argentine), soit à des forts enjeux de conservation (Amazonie, et plus récemment bassin du Congo). Il s'intéresse également aux politiques qui sont menées dans ces régions, et aux concepts et paradigmes qui cadrent ces politiques. C'est pour ces raisons qu'il s'est intéressé à la notion de services écosystémiques, avec laquelle il travaille depuis 2007. Il a notamment coordonné, de 2010 à 2013, un projet de recherche financé par l'Agence nationale de la recherche (Programme Blanc, Appel à projets Jeunes chercheurs/chercheuses) intitulé Approche géographique des services écosystémiques. Ce livre est un des produits de ce projet.

Courriel : xavier.arnauld@cnrs.fr

Catherine Aubertin est économiste de l'environnement, directrice de recherche à l'Institut de recherche pour le développement (IRD). Elle anime plusieurs groupes de recherche sur les politiques d'environnement, le développement durable et l'économie de la biodiversité. Elle travaille au Brésil et en Guyane sur les traductions locales des conventions internationales et l'utilisation des outils économiques pour la conservation (marchés, mécanismes REDD, paiements pour services environnementaux). Elle a publié Protected areas, sustainable land? Ashgate, 2011 (avec E. Rodary); Le développement durable : enjeux politiques, économiques et sociaux. La Documentation française, 2010 (avec F.-D. Vivien); Les marchés de la biodiversité, 2007 (avec F. Pinton et V. Boisvert). Elle est corédactrice en chef de la revue Natures Sciences Sociétés.

Courriel : catherine.aubertin@ird.fr

Originaire de Colombie, Monica Castro a suivi une formation en biologie (Université de Los Andes, Colombie), puis un Master en ethnoécologie (Muséum national d'histoire naturelle, France) et un doctorat en géographie (École des hautes études en sciences sociales, France). Sur cette base fortement interdisciplinaire, Monica développe une approche dite de Political ecology appliquée aux politiques internationales de conservation et gestion des services écosystémiques et leurs effets sur des contextes aussi différents que des espaces agricoles ou des unités de 
conservation dans les pays du Nord (France) et du Sud (Brésil, Gabon). C'est durant le post-doctorat qu'elle a réalisé au sein de l'UMR société environnement territoire (CNRS/Université de Pau et des Pays de l'Adour) qu'elle a mené les recherches qui font l'objet des contributions auxquelles elle participe dans cet ouvrage. Elle effectue, depuis le mois d'octobre 1013, un post-doctorat comme première assistante à l'Institut de géographie et durabilité de l'Université de Lausanne (Suisse).

Courriel : Monica.Castro@unil.ch

Après un doctorat consacré à l'analyse du rôle de Greenpeace et du WWF dans la résolution des problèmes environnementaux (2002), Denis Chartier a mené des travaux sur l'influence des ONG internationales dans les politiques forestières tropicales, du local au global. Il développe actuellement une analyse critique des politiques de développements durables et de conservation de la nature dans les pays du Sud (Brésil Amazonie, Burkina Faso) selon plusieurs orientations théoriques et thématiques. La première examine les différentes visions de la nature que véhiculent les acteurs environnementaux afin d'identifier des espaces et des stratégies d'action en rupture avec la disjonction moderniste nature/ culture. La seconde interroge les théories géographiques et politiques attestant qu'une alliance entre société civile et État-nation permettrait de réinventer des politiques territorialisées multilatérales, multiscalaires et multimétriques, plus adaptées aux processus de mondialisation contemporains. La troisième étudie les modes d'appropriation du concept de service écosystémique et les pratiques de développement dites alternatives, telles que les modes de production agroécologiques ou les réserves extractivistes brésiliennes et interroge leur rôle et leur place dans la construction de politiques de l'anthropocène. Il mène également un travail épistémologique sur l'écologie politique, en particulier dans le champ de la géographie française. Il a été rédacteur en chef de la revue Écologie \& politique de 2007 à 2013.

Courriel :denis.chartier@univ-orleans.fr

Lise Desvallées est agrégée de géographie et élève de l'école normale supérieure de Lyon. Elle réalise une thèse de doctorat sur la précarité énergétique en Espagne et au Portugal au Laboratoire Techniques Territoires et Sociétés (UMR CNRS / Université Paris Est / École des Ponts et Chaussées). Intéressée par la political ecology dès ses travaux de master sur la gestion de l'eau au Maroc puis à Madères, elle a réalisé dans le cadre de l'ANR AGES un stage sur les oppositions aux mécanismes REDD dont est tiré le chapitre qu'elle a écrit dans cet ouvrage.

Courriel : lise.desvallees@ens-lyon.fr 
Simon Dufour est enseignant-chercheur au Département de géographie et d'aménagement de l'espace de l'Université Rennes 2 et membre de l'UMR LETG, équipe COSTEL à l'Université de Rennes 2. Après une formation en écologie, il a réalisé une thèse en géographie sur le fonctionnement et la gestion de la végétation des milieux fluviaux. Depuis, il travaille essentiellement sur les processus et les dynamiques biophysiques qui structurent les hydrosystèmes en mobilisant les approches de l'hydrologie, de la géomorphologie, de la biogéographie et de l'écologie végétale. Pour cela, il utilise et développe des méthodologies variées, depuis les mesures in situ jusqu'aux outils géomatiques. De plus, il s'intéresse aux origines, aux conditions de mise en œuvre et aux impacts effectifs des politiques et des outils de gestion de l'environnement dans les systèmes fluviaux (gestion intégrée, restauration écologique, services écosystémiques, indicateurs, etc.).

Courriel : simon.dufour@univ-rennes2.fr

Michel Grimaldi est agronome et pédologue. Directeur de recherche à l'Institut de recherche pour le développement, il est rattaché à l'Institut d'écologie et des sciences de l'environnement de Paris (IEES-Paris) créé début 2014. Au sein de cette UMR, il est responsable de l'équipe BIOPHYS qui se focalise sur les processus biophysiques de conservation et de dégradation des sols, et leurs implications dans la fourniture de services écosystémiques. Il a travaillé en Guyane et au Brésil où il s'est intéressé aux changements de fonctionnement des écosystèmes amazoniens, consécutifs aux activités agricoles et minières. Il a participé à de nombreuses recherches interdisciplinaires qui ont notamment mis en évidence l'influence de la diversité du sol dans le paysage sur les flux hydrogéochimiques et sur la biodiversité. Dans le cadre du programme de l'Agence nationale de la recherche (Appel à projets 2006 « Biodiversité ») intitulé «Biodiversité des paysages amazoniens ; déterminants socioéconomiques et productions de biens et services écosystémiques » dirigé par Patrick Lavelle (UPMC), il a coordonné le groupe chargé d'évaluer les services écosystémiques; l'approche suivie et les résultats obtenus sont discutés dans les chapitres de cet ouvrage auxquels il est associé.

Courriel : michel.grimaldi@ird.fr

Bernard Hubert est directeur de recherche émérite à l'INRA et directeur d'études à l'EHESS (où il enseigne sur la manière dont la notion de développement durable interpelle la recherche scientifique), Bernard Hubert est depuis 2009 président d'Agropolis International à Montpellier. Après avoir travaillé pendant dix ans à l'Orstom (aujourd'hui IRD) en Afrique de l'Ouest, où il a étudié les dynamiques de population de rongeurs, il a rejoint l'INRA et créé à Avignon l'unité d'Écodéveloppement 
pour étudier les systèmes d'élevage méditerranéen et les questions liées à l'élevage en forêt dans la perspective d'une meilleure prévention des risques d'incendie. Il a ensuite été chef du département Systèmes agraires et développement (SAD) de l'INRA de 1993 à 2003, puis directeur scientifique du secteur Sociétés Économie Décision, jusqu'en 2007. Il a alors créé, à la demande de l'INRA et du CIRAD, le GIP Initiative française pour la recherche agronomique internationale (IFRAI), qu'il a dirigé jusqu'en 2010. Depuis 2010, il préside la CRAI (Commission pour la recherche agronomique internationale) qui regroupe le CIRAD, l'INRA, l'IRD et l'Irstea avec les trois ministères en charge des Affaires étrangères, de la Recherche et de l'Agriculture. Il est l'un des trois rédacteurs en chef de la revue Natures Sciences Sociétés, associate editor de The Rangeland Journal et il préside le comité scientifique de la revue Cahiers Agricultures. Il assume la fonction d'expert pour le développement durable au sein du CIC pour Angkor depuis 2010. Il est membre correspondant de la section Sciences de l'Homme et de la Société de l'académie d'agriculture de France.

Courriel : bernard.hubert@avignon.inra.fr

Olivier Huet a obtenu en 2013 le Master Environnement, Développement, Territoires et Sociétés (cohabilité par le Muséum National d'Histoire Naturelle de Paris et Agroparitech) dans le cadre duquel il a fait un stage de doctorat au Gabon sur les politiques de services écosystémiques. Il s'intéresse plus largement au développement en tant que processus, à la fois dans ses manifestations locales et ses aspects institutionnels et aux questions d'usages des ressources naturelles, notamment aux discours et aux normes qui régissent ces usages.

Courriel : olivierhuet56@gmail.com

Nicolas Jégou est docteur en mathématiques appliquées. Il enseigne probabilités et statistiques à l'Université Rennes 2. Il intervient notamment dans le département Géographie, en Licence et en Master. Il a collaboré à l'écriture de plusieurs livres concernant l'utilisation du logiciel $\mathrm{R}$ en statistique. Ses travaux de recherche portent essentiellement sur la régression non paramétrique. Ceux-ci concernent en particulier les modèles additifs et la régression sous contraintes de formes.

Courriel : nicolas.jegou@univ-rennes2.fr

Christian Kull est géographe et political ecologist. Enseignant chercheur à l'université de Monash (Melbourne, Australie) au moment de la rédaction de cet ouvrage, il a été nommé depuis au département de géographie de l'Université de Lausanne. Ses recherches portent sur les conflits et les débats autour de la gestion des ressources naturelles, en 
particulier sur des sujets comme les feux de brousse, les aires protégées, les plantes exotiques et les forêts. Il est auteur de nombreux articles et du livre Isle of Fire : the Political Ecology of Landscape Burning in Madagascar (University of Chicago Press, 2004).

Courriel : christian.kull@monash.edu

Solen Le Clec'h est doctorante en géographie au sein de l'UMR LETG, équipe COSTEL à l'Université de Rennes 2. Elle est coordinatrice du Pôle Brésil - São Paulo de l'Institut des Amériques (IDA). Elle a commencé en 2012 une thèse de doctorat intitulée La spatialisation des services écosystémiques : quel apport à la gestion des milieux en contexte de front pionnier (Contrat doctoral IDA/MESR). Elle s'intéresse plus précisément à la spatialisation des processus physiques qui induisent des services écosystémiques et au lien entre les processus à l'origine de la fourniture des services écosystémiques, les politiques publiques et les activités anthropiques qui s'opèrent sur un territoire.

Courriel : solen.leclech@uhb.fr

Noël Ovono Edzang est enseignant-chercheur au Département de Géographie de l'Université Omar Bongo. Il a soutenu en 2001 un doctorat de Géographie tropicale à l'Université Bordeaux 3. Après six années passées à l'Institut de recherche en sciences humaines (IRSH) du CENAREST (Libreville-Gabon), il intègre comme Enseignant permanent en novembre 2007, le Département de géographie de l'Université Omar Bongo (Gabon) et le Centre d'études et de recherche en géosciences politiques et prospective (CERGEP). Rédacteur en chef de la revue Gabonica, il est depuis juin 2011, il est le coordonnateur (composante Gabon) du Master Professionnel et de Recherche conjoint entre l'Université Omar Bongo et l'Université de Yaoundé II Soa (Cameroun) intitulé « Dynamiques, Gestion et Sécurité des Espaces Transfrontaliers » (DGSET). Depuis octobre 2013, il est en outre directeur de la Coopération universitaire à l'Université Omar Bongo. Auteur de différents articles scientifiques et d'un ouvrage, il mène actuellement des recherches qui portent sur la gouvernance foncière, le développement rural et le développement local au Gabon et dans les pays de la CEMAC.

Courriel : noel_ovono@yahoo.fr

Johan Oszwald est enseignant-chercheur au Département de géographie et d'aménagement de l'espace de l'Université Rennes 2 et membre de l'UMR LETG, équipe COSTEL (Université de Rennes 2) et de l'Équipe d'accueil ECODIV (Université de Rouen). Johan Oszwald a réalisé une thèse en géographie sur la cartographie des fronts de déforestation en Côte d'Ivoire entre 2002 et 2005. Depuis, il travaille 
essentiellement sur les interactions hommes / milieux en situation de front pionnier tropical de déforestation ou dans des espaces fortement impactés par les activités humaines.

Courriel : johan.oszwald@univ-rennes2.fr

Leticia Sello Madoungou est docteure en géographie. Après avoir fait sa licence à l'Université Omar Bongo (Gabon) et son master à l'université Bordeaux 3, elle a, en décembre 2013, soutenu à l'Université de Pau et des Pays de l'Adour une thèse de doctorat intitulée Le monde rural gabonais, entre production et conservation. Ses travaux portent sur les rapports entre les populations rurales et leur environnement, et plus particulièrement sur les rapports entre sécurité alimentaire, parcs nationaux et développement local.

\section{Courriel : 1sellomadoungou@yahoo.fr}

Iran Veiga a une formation d'ingénieur agronome et un doctorat en sociologie rurale. Son travail de recherche porte sur les interfaces sociales créées par les interventions de développement en milieu rural, et particulièrement par les rapports entre les savoirs locaux et les savoirs scientifiques. Il a développé ces thèmes dans les régions de frontière agraire de l'Amazonie orientale brésilienne et, plus récemment, en Argentine et en France, où ses travaux se centrent sur la transformation des savoirs et des compétences des agents de développement en milieu rural face à de multiples situations et interfaces de développement. Entre 1996 et 2013, il a travaillé comme enseignant-chercheur au Département de sciences agraires et développement rural de l'Université fédérale du Pará (à Belém, Brésil), dans une équipe pluridisciplinaire de recherche et d'enseignement sur l'agriculture familiale, les populations traditionnelles et le développement rural, ainsi que, entre 2007 et 2013, au Laboratoire Agriterris, plateforme qui regroupe des institutions de formation et de recherche argentines, françaises et brésiliennes travaillant sur le développement rural. En 2014, il est basé à Paris, où il développe ses activités de recherche à l'INRA (Institut national de la recherche agronomique) dans l'unité SenS (Sciences en Société), une équipe pluridisciplinaire de recherche, d'enseignement et d'expertise travaillant sur les relations entre sciences, techniques et sociétés.

Courriel : veiga.iran@versailles.inra.fr 